\title{
Tongue laceration during electroconvulsive therapy
}

\author{
Sang-Woo Woo ${ }^{1}$, and Sang-Hwan Do $^{2}$ \\ Department of Anesthesiology and Pain Medicine, ${ }^{1}$ Seoul National University Hospital, Seoul, ${ }^{2}$ Seoul National University Bundang \\ Hospital, Seongnam, Korea
}

Electroconvulsive therapy (ECT) had been used for psychiatric diseases such as depression. Recently, modified ECT is preferred, where the patient is in a state of muscle relaxation induced by muscle relaxant before the influx of electrical current.

Although muscle relaxant is used, forceful jaw clenching is inevitable during ECT, since masticatory muscles, particularly the temporalis and the masseter, are directly stimulated by the electrical current. Although biting pressure in a conscious individual is usually controlled by a feedback mechanism to avoid excessive strain, this feedback mechanism does not come into action in an anesthetized patient [1]. Therefore, the teeth or other intraoral structures can be injured from biting or invagination during modified ECT.

Psychiatric patients are especially vulnerable to intraoral injuries during modified ECT, because they are more likely to have drug-related dry mouth, smoking, and/or alcohol addiction. In xerostomia patients, the change in the $\mathrm{pH}$ and the immune function secondary to reduced salivary flow may also lead to an increased risk of intraoral infection [2].

In order to prevent intraoral injury during modified ECT, mouthguards rather than oral airways are used. Various types of gags and mouthguards have been used such as rubber bite blocks, braided guaze gags, gauze wrapped tongue blades and mouthguards. McClure devised a gag made of dental plastic, and also described about molded mouthguards [1]. But individually molded mouthguards like hard rubber bite blocks are so firm that care should be given to prevent soft tissue injuries such as tongue bites which can occur between the teeth and the mouthguard.
We would like to describe a tongue laceration case which occurred during modified ECT despite using a mouthguard. A 71-year-old female patient was admitted for modified ECT under the diagnosis of depression. The mouthguard was made of silicone, immersed in hot water before use and transformed to fit the denture of the patient (Fig. 1A). After modified ECT was given, tongue laceration was found, which seemed to have occurred from biting between the mouthguard and the teeth. Tongue laceration was $1.5 \mathrm{~cm}$ long and $0.5 \mathrm{~cm}$ deep. Neither active bleeding nor hematoma was observed. After physical examination by an otolaryngologist, it was sutured simply with Vicryl 4-0 under local anesthesia (Fig. 1B). The wound recovered well without complication.

Intraoral soft tissue injuries, especially tongue injuries generally recover well after simple treatment because of the abundant blood supply. Most tongue lacerations do not require sutures. Lacerations that should be repaired with suture include bisecting wounds, lateral border wounds, wounds with active hemorrhage, wounds larger than $2 \mathrm{~cm}$, gaping wounds and amputation injuries $[3,4]$. If the patient cooperates well, tongue laceration can be sutured under local anesthesia using absorbable material such as Vicryl 4-0 [3].

Some lacerations may cause airway complications including intraoral structure swelling and difficult breathing. In the case of hematoma, blood fills the submandibular space, the floor of the mouth becomes full, and then the tongue is forced upwards and backwards, causing an elevation of the tongue and airway obstruction. If swelling and difficult breathing became severe, early nasotracheal intubation or tracheostomy should be considered for airway protection. Extubation should

Corresponding author: Sang-Hwan Do, M.D., Ph.D., Department of Anesthesiology and Pain Medicine, Seoul National University Bundang Hospital, 166, Gumi-ro, Bundang-gu, Seongnam 463-707, Korea. Tel: 82-31-787-7501, Fax: 82-31-787-4063, E-mail: shdo@snu.ac.kr (c) This is an open-access article distributed under the terms of the Creative Commons Attribution Non-Commercial License (http:// creativecommons.org/licenses/by-nc/3.0/), which permits unrestricted non-commercial use, distribution, and reproduction in any medium, provided the original work is properly cited. 

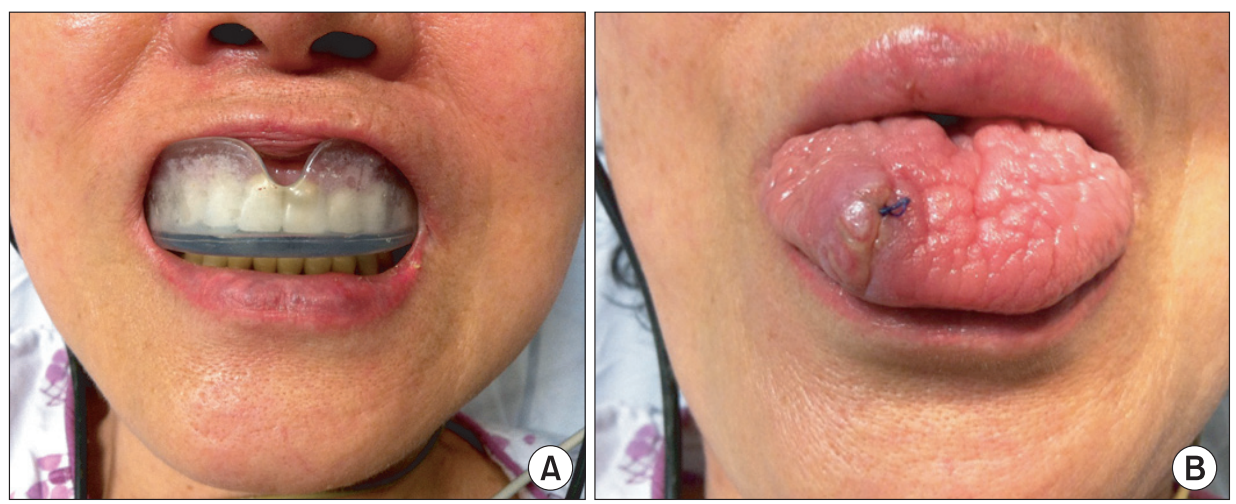

Fig. 1. The mouthguard in proper position (A). Tongue laceration repaired with a simple continuous suture. The tongue has an irregular and dry fissured surface, which is a typical characteristic of dry mouth (B).

be performed after the swelling subsides. While active arterial bleeding requires intervention, subsequent treatments are usually conservative, such as steroids, antibiotics and reversal of any coagulopathy [5].

Before all else, one should examine the patient's dental and oral condition carefully and check whether the mouthguard fits well to the patient before performing ECT. If necessary, the patient should get dental treatment in advance to ensure his or her safety during ECT.

\section{References}

1. Kiran S, Bala R, Singh T. Dental protection during modified electro- convulsive therapy using roll-gauze mouth gag. J ECT 2009; 25: 74-5. 2. Johnson R, Johnson G. Integrating palliative and curative care strategies in the practice of otolaryngology. Flint : Cummings Otolaryngology: Head \& Neck Surgery. 5th ed. Philadelphia, Mosby. 2010, pp 1102-3.

3. Patel A. Tongue lacerations. Br Dent J 2008; 204: 355.

4. Brown DJ, Jaffe JE, Henson JK. Advanced laceration management. Emerg Med Clin North Am 2007; 25: 83-99.

5. Lo BM, Campbell BH. A traumatic swollen tongue. Resuscitation 2010; 81: 267. 